\title{
PROCEEDINGS REPRINT
}

(10) SPIE-The International Society for Optical Engineering

\author{
Reprinted from
}

$c$

\section{Videometrics III}

2-4 November 1994

Boston, Massachusetts

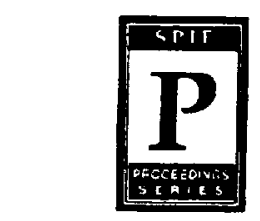

Volume 2350 


\title{
Mode shape analysis using a commercially available "peak store" video frame buffer
}

\author{
Walter L. Snow and Brooks A. Childers \\ NASA Langley Research Center MS 236 \\ Hampton, VA 23681-0001 \\ Tel.: (804) 864-4612,Fax: (804) 864-7607, e-mail: w.l.snow@larc.nasa.gov,b.a.childers@larc.nasa.gov
}

\begin{abstract}
Time exposure photography, sometimes coupled with strobe illumination, is an accepted method for motion analysis that bypasses frame by frame analysis and re synthesis of data. Garden variety video cameras can now exploit this technique using a unique frame buffer that is a non integrating memory that compares incoming data with that already stored. The device continuously outputs an analog video signal of the stored contents which can then be redigitized and analyzed using conventional equipment.

Historically, photographic time exposures have been used to record the displacement envelope of harmonically oscillating structures to show mode shape. Mode shape analysis is crucial, for example, in aeroelastic testing of wind tumnel models. Aerodynamic, inertial, and elastic forces can couple together leading to catastrophic failure of a poorly designed aircraft. This paper will explore the usefulness of the peak store device as a videometric tool and in particular discuss methods for analyzing a targeted vibrating plate using the "peak store" in conjunction with calibration methods familiar to the close-range videometry community. Results for the first three normal modes will be presented.
\end{abstract}

Keywords: modal analysis, frame buffer, close-range photogrammetry, calibration, motion analysis

\section{INTRODUCTION}

Anyone who has viewed footage of the Tacoma Narrows Bridge collapse will appreciate the importance of aeroelasticity. This structure, designed to withstand $100 \mathrm{mph}$ winds, failed at a wind speed of $42 \mathrm{mph}$ due to a flutter instability. ${ }^{1}$ Flutter is an oscillatory instability which occurs when an accidental flow or structural disturbance couples with one or more structural resonances and allows the structure to extract energy from the flow in destructive proportions. Aeroelastic instability prediction for complicated aircraft is proceeding rapidly but careful experimental testing of scaled models in specialized wind tunnels is essential to successful and safe designs. The experimental determination of the eigenfrequencies and modes of a complicated model is often an essential preliminary step in testing. Photographic time exposures of models excited at discrete frequencies have been used in the past for mode mapping. This paper describes an unusual frame buffer which allows time exposures using standard video cameras. Close range videogrammetry techniques are used to quantify the measurements. The device is useful for visualizing and measuring the generalized coordinates of some structures as well as the influence functions which govern their deformation.

\section{2. "PEAK STORE" CONCEPT}

Occasionally an idea bursts upon the scene whose simplicity belies its cleverness and assures its impact on future development. The video peak store is one example which combines video with single purpose image-processing capability to allow an unusual mode of time integration which might best be termed time information. The concept is depicted in Fig. 1. The input and output to the device is analog composite video so the unit can be considered to be an intelligent buffer in the video line. The analog input is AVD converted and stored to memory when the device is activated. All subsequent frames are mediated by a high speed comparator loop between the digital input and output of memory. If the input level exceeds what is already stored, then the memory contents are over written with the new information,

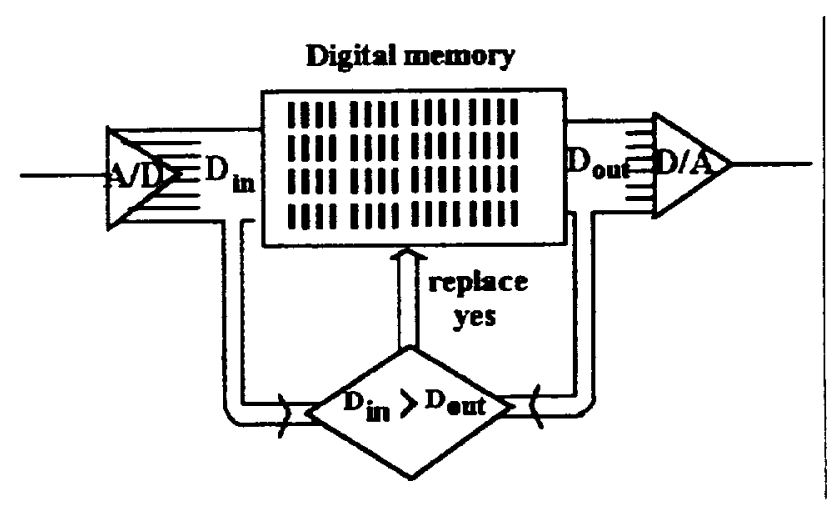

Fig. 1. Simple block diagram of peak store concept. 
otherwise not. The memory contents are continually D/A converted and packaged as composite video output suitable for display on a monitor, stored on tape or disk, or frame grabbed by computer. A photograph of the unit is shown in Fig. 2. Video level and pedestal are adjustable and the logic circuitry can be bypassed using the normal mode. Attention is drawn to the toggled black/white mode which is useful when one wishes to replace the memory contents only when the input is less than what is stored. This is ideal for detecting motion of dark objects against a light background. The device has been found useful for teaching motion analysis and optics

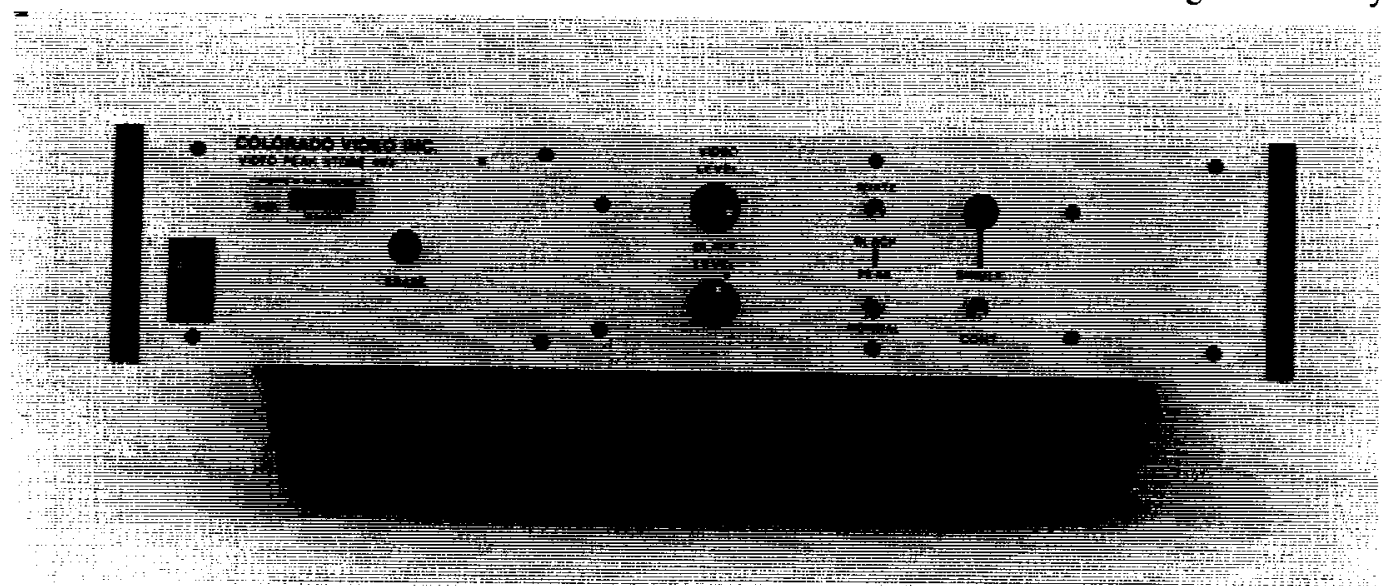

Fig. 2. Model 493 video peak store control panel.

concepts ${ }^{2}$ and for photomacrography ${ }^{3}$. The articles by Southworth ${ }^{4567}$ scope a number of other useful applications.

\subsection{Tracking examples.}

The peak store device compresses temporal information and is an excellent diagnostic tool for applications work. The analog input to the peak store is any composite video signal. Although a camera is one possibility, more frequently it is a tape or video recorder. The stored media playback is preferable in most cases not only because of post editing ease but also because the live video image memory can inadvertently be erased by light loss in black mode or 'whited out' by a light flash in white mode.

A significant problem in motion analysis studies is automated tracking of targets in an image sequence to avoid having to manually identify targets on a field by field basis. One pixel flow estimator fits previous centroid information to low order polynomials and extrapolates to the next time step. This works nicely when the motion is benign as in the airplane case depicted in Fig. 3 and reported
in earlier work.

This figure entails four seconds of data with each subimage separated by 10 frame [ $1 / 3$ seconds] intervals. For most of this study frame analysis was possible with zero ${ }^{\text {th }}$ order prediction i.e. the motion could be tracked by simply updating the centroid window. This was not the case for the tethered marble imaged in the companion photo. This case was used to evaluate predictions for tracking in a more demanding application. ${ }^{9}$ Here the fields are separated by $1 / 60$ seconds and the loss of one dimension in projection precludes successful 
use of polynomial extrapolation. The peak store device allows simple evaluation of tracking scenarios which otherwise would require
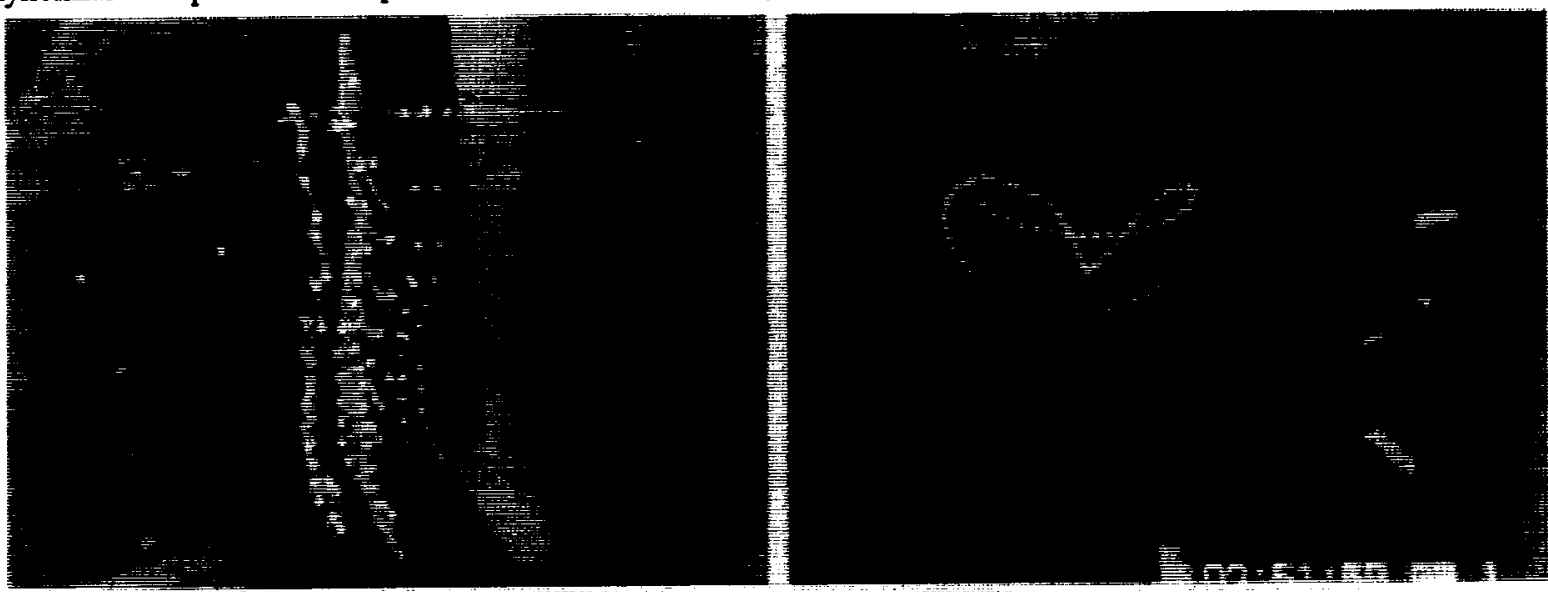

Fig. 3. Pixel flow. Tethered model airplane at 10 frame intervals and pendulum at video field rate intervals.

measurements of dozens of images and subsequent replotting to display meaningful information.

\subsection{Videometric calibration.}

While the peak store device is a tool for the visual display of information it can be used for quantitative measurement as well. The asdelivered module is set up for RS-170 one volt peak to peak composite video with 2:1 interlace with other customized options available. The internal frame store has $512 \times 480$ elements but is transparent to the user. Analog waveform comparisons of individual input and output video lines using a digitizing oscilloscope were indistinguishable but for slight de offsets which were somewhat adjustable using front panel controls.

To assess the impact of introducing the peak store in the video acquisition link, a laboratory close range photogrammetry test range was used ${ }^{10}$.The imaging geometry is depicted in Fig. 4. The camera was a Cohu $4990 \mathrm{CCD}$ camera having $768 \mathrm{H} \times 494 \mathrm{~V}, 8.4 \times 9.8 \mu \mathrm{m}$ cells arranged on a $6.4 \times 4.8 \mathrm{~mm}$ active area. The imager is listed as a $\mathrm{HAD}$ interline transfer $\mathrm{CCD}$ with on-chip microlenses. For these tests the camera was equipped with a $12.5 \mathrm{~mm} f / 1.3$ lens. A photogrammetric network analysis program ${ }^{11}$ was used to assess the system. Network adjustment was computed using a 16 station network of camera locations in a conical pattern around a target plate and at two elevations. Convergence angles of diametrically opposed camera stations directed at the center of the target plate were approximately 78 and 85 degrees for the two elevations. The camera was rolled about the optical axis in order to minimize correlation between parameters in the adjustment. The target plate was an array of $11 \times 11$ diffuse paper targets arranged in a nominal 2 inch spacing on a black background.

At each camera configuration an image was taken with the peak store in the acquisition loop and a companion image acquired within a few minutes by coupling the input and output cables together with a barrel connector. The video in either case was frame grabbed into a PC using a $752 \times 480$ pixel format. The results of the adjustments are listed in the table.

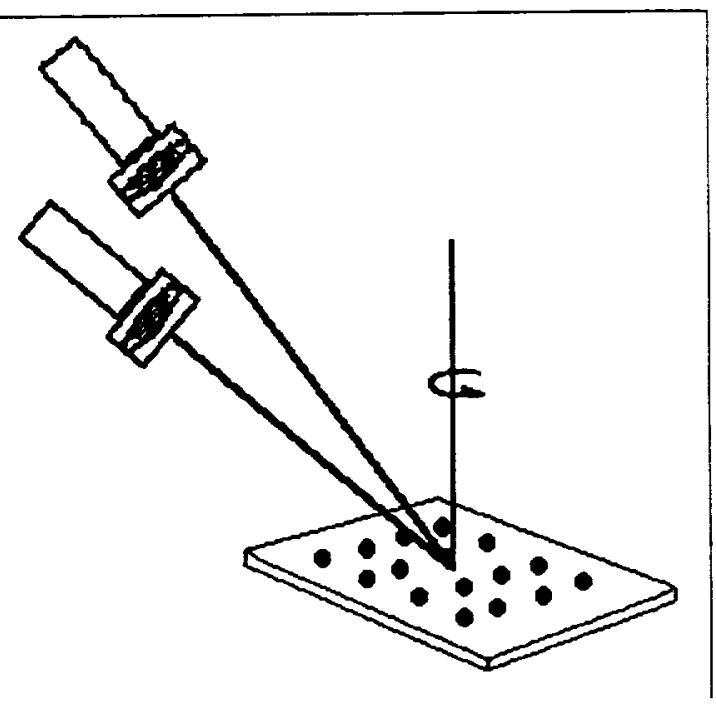

Fig. 4. Network geometry. 


\begin{tabular}{|l|c|c|c|c|}
\hline Case & $\sigma_{0}$ & $\begin{array}{c}\text { mean } \\
\text { image } \\
\text { precision } \\
\text { [microns] }\end{array}$ & $\begin{array}{c}\text { mean } \\
\text { object } \\
\text { precision } \\
\text { [mils] }\end{array}$ & $\begin{array}{c}\text { relative } \\
\text { precision }\end{array}$ \\
\hline with peak store & 1.01 & 0.25 & 0.48 & 58000 \\
\hline without peak store & 1.02 & 0.30 & 0.58 & 48000 \\
\hline
\end{tabular}

Table 1. Results of photogrammetric network adjustment. $\sigma_{0}$ is unit weight precision.

There is no indicated degradation and in fact for static scenes the averaging tends to smooth the noise while adding a fixed brightness level as initially pointed out by Southworth ${ }^{6}$. The constant background is removed by thresholding for videometry so is inconsequential.

\section{MODAL ANALYSIS}

The description of complicated motion can be simplified considerably if described in terms of the eigenfunctions of the governing equations. Linear combinations of the eigenfunctions belonging to the dominant frequencies suffice to characterize otherwise hopelessly complicated situations. The orthogonality ${ }^{12}$ properties of the characteristic functions are useful for analysis. The eigenfunctions for vibration problems are of course the normal modes. Only in simplest circumstances are they obtainable from first principles. In practice accelerometers or other types of pick-ups are used to determine mode shapes. Care must be taken so that the mass of the measurement device does not bias the measured property. Photographic time exposures ${ }^{13}$ have been used for non contact measurements of mode shapes. The peak store device provides an electronic version of the photographic approach which is much more convenient to use.

To demonstrate the technique, a $24^{\prime \prime} \times 48^{\prime \prime} \times 0.125^{\prime \prime}$ piece of scrap aluminum was chosen as a test article and clamped to an optics table. The gauge of the metal was chosen so that the natural frequencies of the object are in the $10 \mathrm{~Hz}$ range and commensurate with those found in thin wing aeroelasticity studies. The experimental layout is sketched in Fig. 5. The cameras are Pulnix 2/3 inch format TM-845 frame transfer devices with $11.5 \mu(H) \times 13.5 \mu(V)$ sensor elements. The cameras were equipped with $12.5 \mathrm{~mm}$ focal length lenses and placed in opposition to one another far enough away to encompass the entire test object. The placement is a compromise between maximum sensitivity to displacement i.e. in the plane of the object and the desire to obtain optimum triangulation accuracy viz., 90 degree intersection cones. The included angles for the camera axes were on the order of 75 degrees which for the two camera configuration resulted in triangulation accuracy on the order of 10 to $15 \mathrm{mils}$. [1 mil $=0.001 \mathrm{l}$ ] To establish the eigenfrequencies of the aluminum sheet a photonic sensor was used to monitor the time history of the displacement due to

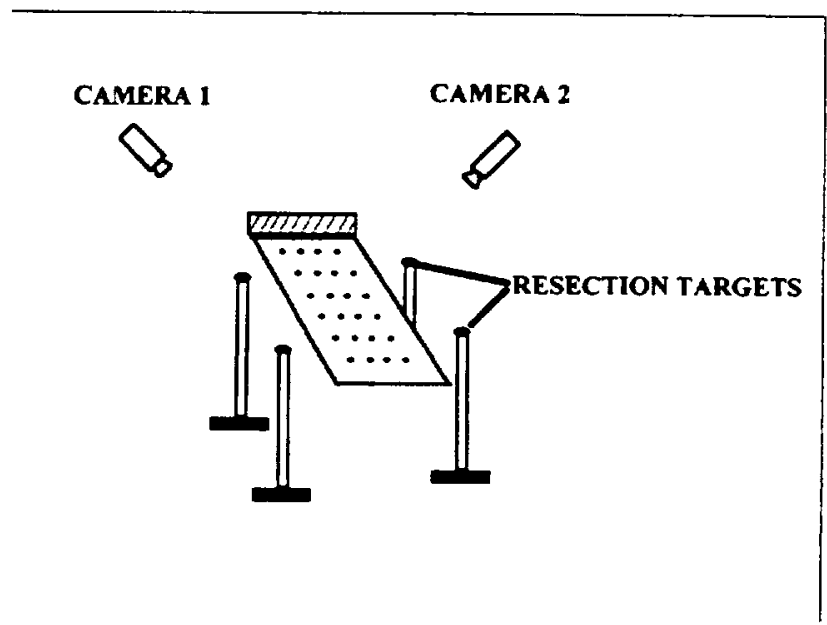

Fig. 5. Measurement geometry. an impact force generated by a small hammer blow. The optical displacement sensor projects a beam of light from a semiconductor laser onto the measurement surface. An aspheric lens collects the scattered bearn and focusses it onto a linear array. The sensor was a Keyance LB- 70 with a $3 \mathrm{~dB}$ response bandwidth of $700 \mathrm{~Hz}$ and so could easily detect non-aliased frequencies for the test object. A schematic of the set up is shown as an insert to Fig. 6 which depicts the amplitude response for the impact. The structural damping is clearly evident in the waveform as is the multi frequency composition of the signal A Fourier transform of the amplitude, shown also as an insert, clearly identifies three frequencies. The digitizer sampling rate used to acquire the input for the FFT was $2 \mathrm{kHz}$. No frequencies within the bandwidth of the sensor were evident beyond $15 \mathrm{~Hz}$.

Having established where the characteristic frequencies were it remained but to excite them to record the amplitude envelope. This was done using a Ling Electronics model 420 air cooled shaker. The shaker has a force rating of 100 pounds, a frequency range of DC 


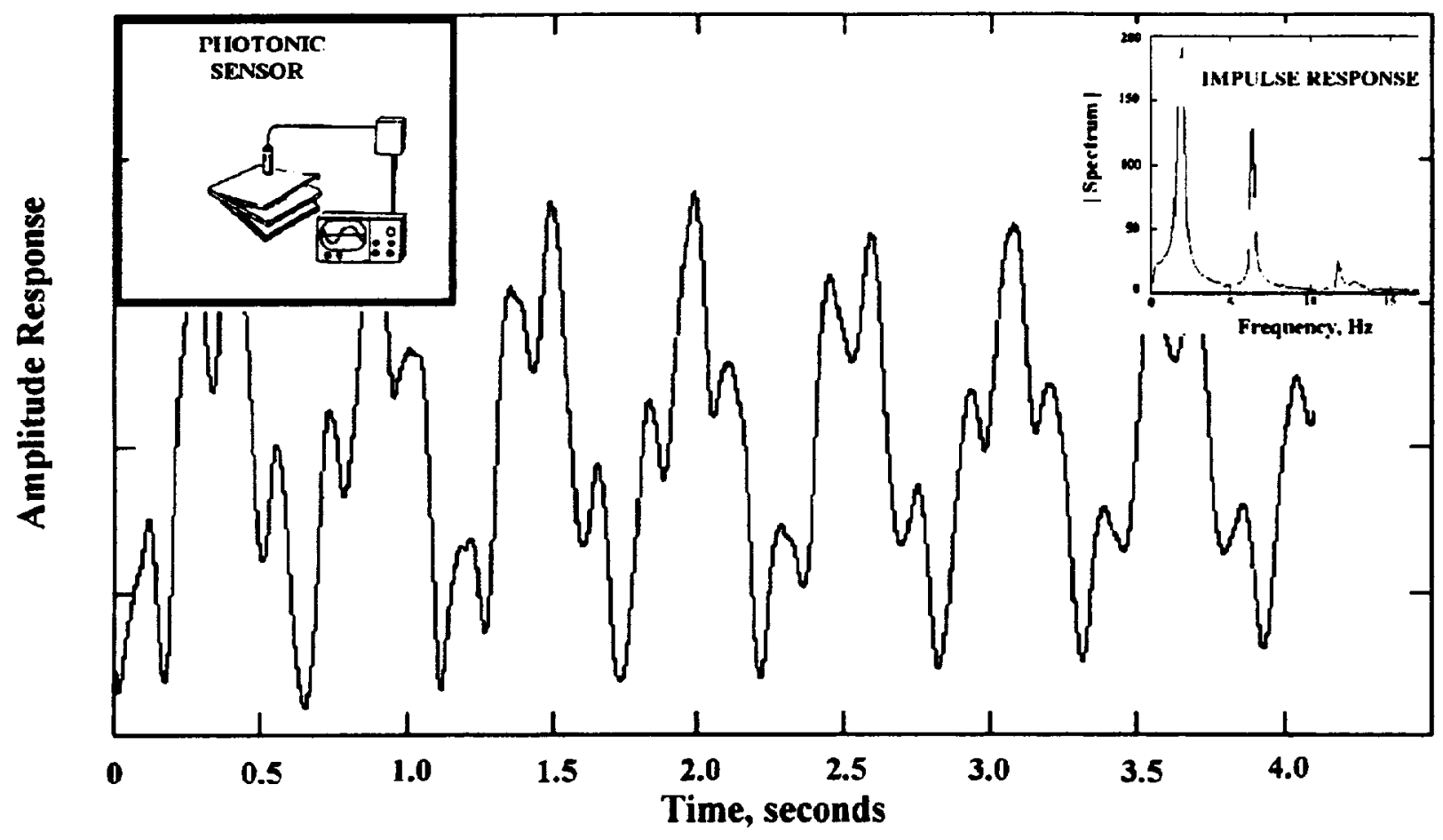

Fig. 6. Amplitude and spectral response using a photonic sensor.

to $7.5 \mathrm{kHz}$ and an armature displacement of \pm 0.375 inches. The plunger appears as a shiny rod barely visible beneath the plate in subsequent images. The images were Vertical Interval Time Coded [VTTC] and subsequently played back through the peak store module to acquire the photo records. The views from camera one [Note the second line of user bits in the image labels.] are shown in Fig. 7. The over writing of time code is evident from the garbled digits of time code. The first longitudinal mode is evident in the top left. The first torsional mode at $6.35 \mathrm{~Hz}$ in the upper right and the second longitudinal mode in the lower right quadrant at $11.70 \mathrm{~Hz}$ with its amplitude diminished in accordance with inverse frequency squared.

Paired composite images as shown in Fig. 8 can be used to quantify the excursions using triangulation. For the first longitudinal mode depicted in the figure the excursion of the end point in the top corner was determined to be \pm 1.62 inches. The peak store provides the researcher with a powerful $3 \mathrm{D}$ histogram rendition of the shape information which is quantifiable. The alternative is to process several hundred image pairs, analyze the multi dimentisonal excursions and re plot them in perspective view. The field by field analysis of time stamped imagery is significant for non steady state cases when phase information is important. Indeed it is the phase interplay between different modes which causes runaway instability. The peak store results are a quantitative adjunct to, not a replacement for, motion analysis. 


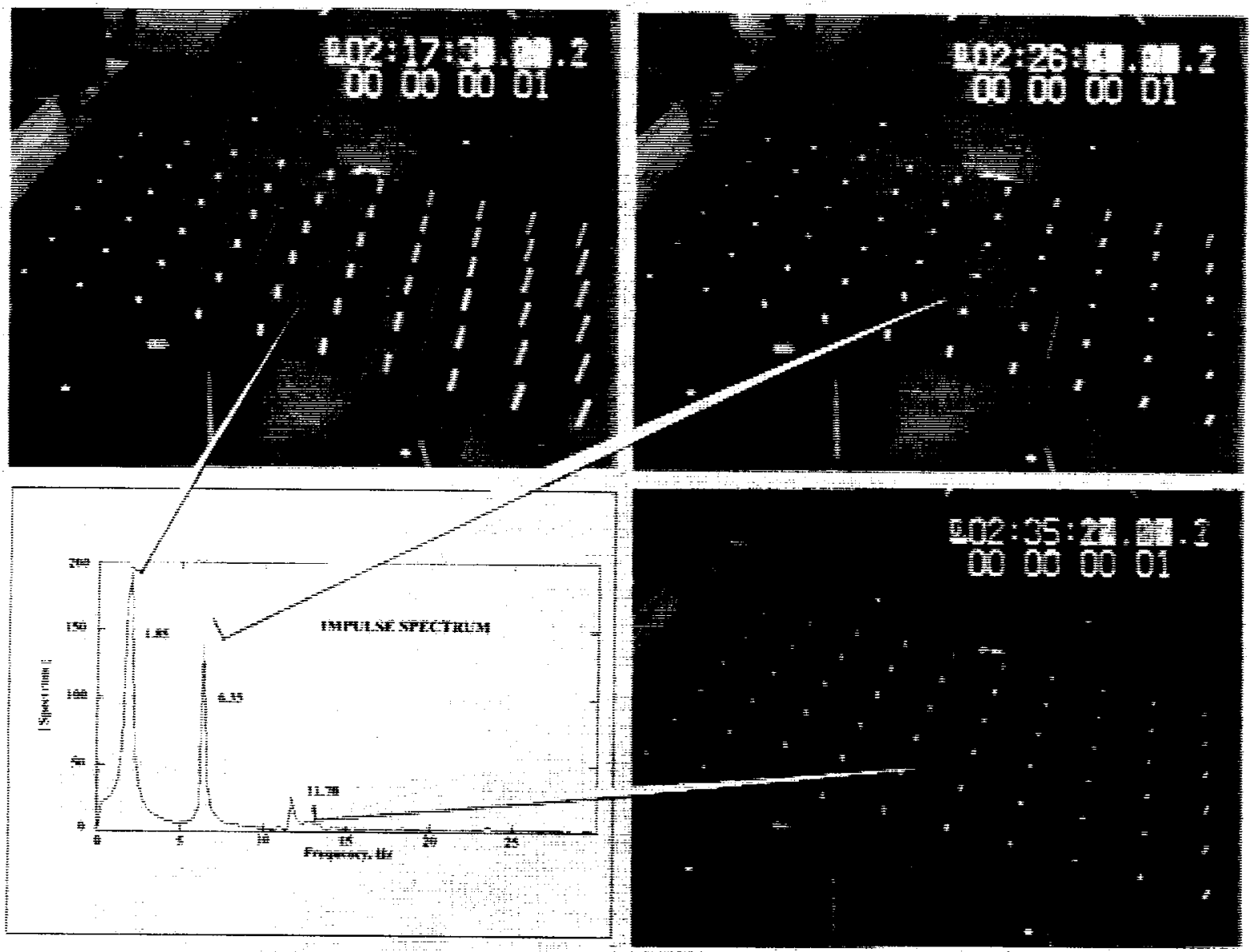

Fig. 7 Spectrum and peak view renditions of modal structure.
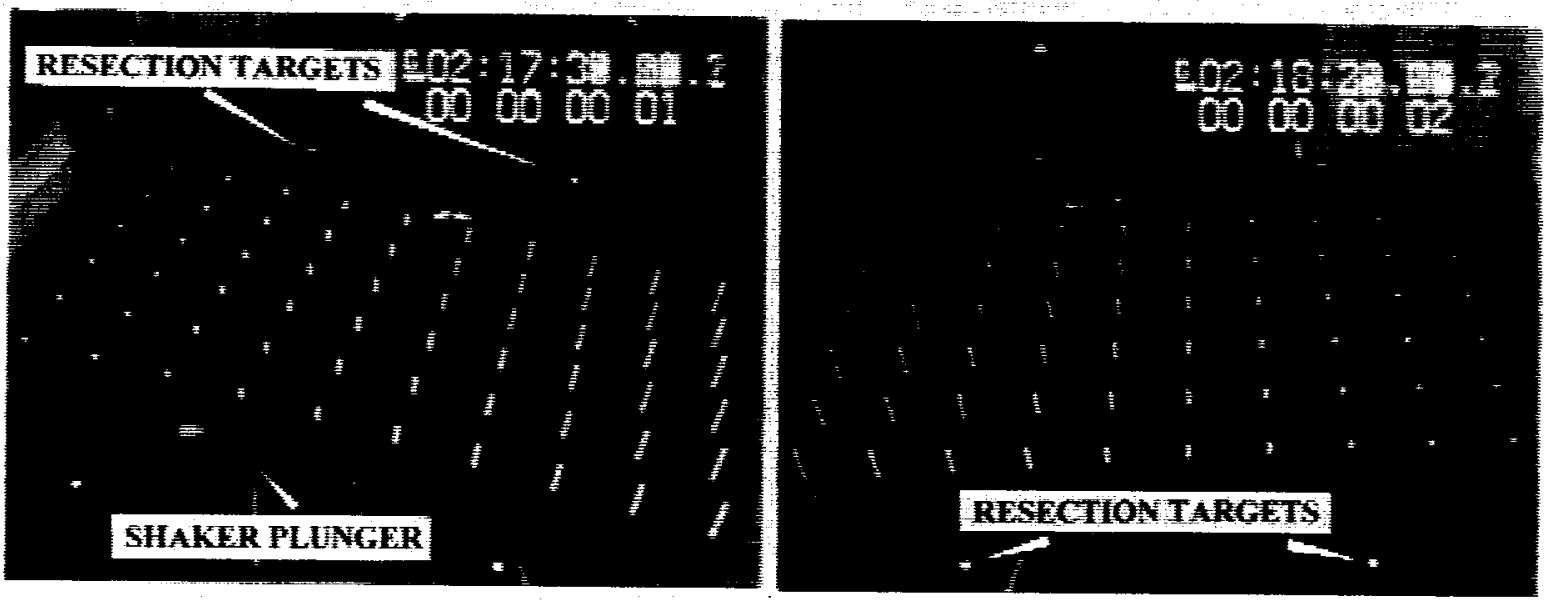

Fig. 8. Paired views of first longitutinal mode using the peak store buffer. 


\section{INFLUENCE MATRICES}

If a structure is deformed within its elastic limits, then it can be characterized completely by its influence functions or the discretized counterparts - the flexibility influence coefficients. If $q$ is a generalized deflection corresponding to a generalized force $Q$ then, using summation convention,

$$
q_{i}=c_{v} Q_{t} \quad t_{i} j=1, \ldots n
$$

i.e. the total deflection is a linear combination of the loads applied over the $\mathrm{n}$ locations scaled by the appropriate matrix element. The matrix equation can be inverted to provide forces as a linear combination of displacements.

$$
Q_{1}=K_{v} q_{t}
$$

where $\boldsymbol{K}_{u j}$ is the stiffness-influence coefficient and identical to the spring constant for the one dimensional case. Energy arguments can be invoked to show that $\{c\}$ and $\{\mathrm{K}\}$ are symmetric. Photo triangulation can be used to determine the deflection due to applied loads and hence determine the flexibility coefficient matrix. This is potentially a large array of numbers and difficult to envision. The peak store device can be used to visualize (as well as measure) the global deflection corresponding to point loads. Fig. 9 shows the effects of a five pound-force load applied to two different locations on a $10 \times 6$ array of load points. Since these are static
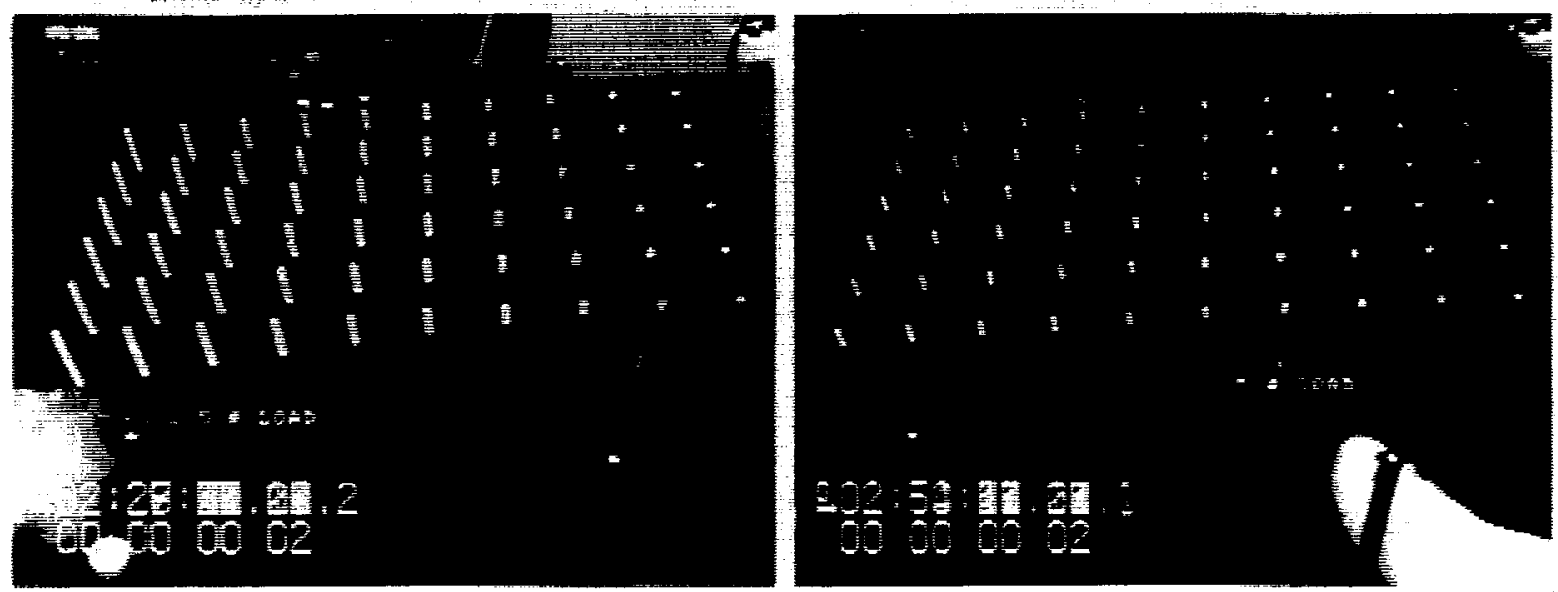

Fig. 9. Deflections due to two point loads.

measurements, the data can also be acquired from 60 snapshot frame pairs with the peak store in normal mode. Notice that a force along the gravity axis induces displacement along other orthogonal axes in compliance with the constraints of the model.

\section{CONCLUSIONS}

A recently released intelligent frame store device is a useful adjunct to the videometrists tool kit which enables time exposure shots of video scenes hitherto available only with film.

No videometric degradation was noted when the unit was examined with bundle adjustment techniques.

The unit is useful for quantitatively compressing video imagery for motion analysis, mode shape, and tracking applications. 


\section{REFERENCES}

1. Y. C. Fung, An Introduction to the Theory of Aeroelasticity, Dover Publications, Inc., 31 East 2nd Street, Mineola, N.Y. 11501, 1993.

2. Robert R. Cadmus Jr., "A video technique to facilitate the visualization of physical phenomena," American Journal of Physics 58(4), pp. 397-399., April, 1990.

3. Andrew Davidhazy, "Light scanning photomacrography with electronic memory unit," Electronic Imaging, pp. 8-9, January, 1994.

4. Glenn Southworth, "Digital processing enhances time exposure in difficult light," Electronic Imaging, p. 5, January, 1992.

5. Glenn Southworth, "Video time exposures for displacement measurement," Lasers \& Optronics, pp. 65-66,

September, 1991.

6. Glenn Southworth, "Time exposures boost sensitivity," Lasers \& Optronics, pp. 17-18, October, 1992.

7. Glenn Southworth, "Video display of temporal phenomena," SPIE Vol. 1820 Videometrics, Boston, (1992) pp. 93. 102 .

8. B. A. Childers, W. L. Snow, S. B. Jones, J. M. Franke, and M. R. Shortis, "Support of wake vortex detection research in flight and wind tunnel testing using videometric techniques, ISPRS Commission V Intercongress Symposium, March 1-4, 1994, Melbourne, Australia, pp. 41-46.

9. Walter. L. Snow, Brooks A. Childers, Stephen B. Jones, and Charles M. Fremaux, "Recent experiences with implementing a video based six-degree-of-freedom measurement system for airplane models in a 20 foot diameter vertical spin tunnel", SPIE Vol. 1820, Videometrics, November 15-16, 1992, Boston, Massachusetts, pp. 158-180.

10. Mark R. Shortis, Walt L. Snow, Brooks A. Childers, and William K. Goad, "The influence of storage media on the accuracy and repeatability of photogrammetric measurements using CCD cameras," SPIE Vol. 2067, Videometrics II, September 9-10, 1993, Boston, Massachusetts, pp. 80-92

11. CRAMPA Close Range Multistation Photogrammetric Adjustment program, Geometric Software, 17 Toxteth Park Street, Coburg 3058, Australia.

12. Raymond L. Bisplinghoff, Holt Ashley, and Robert L. Halfman, Aeroelasticity, Addison-Wesley Publishing Company Inc., Reading, Massachusetts, 1955.

13. L. H. Ohman and M. C. van Wijk," A photogrammetric method of determining the mode shapes of vibrating objects, and results for a thin wing." National Research Council of Canada, Aero. Report \#LR-362, NRC No. 7210 , November, 1962. 


